\title{
SC-FDMA -AN EFFICIENT TECHNIQUE FOR PAPR REDUCTION IN UPLINK COMMUNICATION SYSTEMS -A SURVEY
}

\author{
Prittu Ann Thomas ${ }^{1}$, M. Mathurakani ${ }^{2}$ \\ ${ }^{1}$ PG Student [Wireless Technology], ${ }^{2}$ Professor, Dept of ECE, Toc-H Institute of Science and Technology, Kerala, India
}

\begin{abstract}
In this paper, a survey on different modulation schemes having lowest peak to average power ratio (PAPR) value for use in uplink communication has been studied. OFDM has become the popular modulation choice for high data rate wireless communication systems. It can provide multi-path delay spread tolerance and is robust to channel dispersions. But one of the major limitations of OFDM is the large variation in signal amplitude which gives it a high PAPR. OFDM signals with high PAPR will suffer from nonlinear distortion due to non-ideal behavior of High Power amplifiers as well as it is detrimental to battery powered devices like mobile phones which are power limited.SC-FDMA was found to have a better PAPR reduction than OFDMA and has become modulation choice for uplink communication in Long Term Evolution (LTE).
\end{abstract}

Keywords: OFDM, PAPR, SC-FDMA, Localized FDMA, Interleaved FDMA, SC-FDMA-CDMA

\section{INTRODUCTION}

The demand for a radio system with high data rate and throughput has been increasing tremendously in recent years. To support these requirements third generation partnership project (3GPP) has developed a new radio access technology called long term Evolution (LTE). LTE can provide a data rate of $100 \mathrm{Mbps}$ in downlink and $50 \mathrm{Mbps}$ in uplink when operating in a bandwidth of 20Mhz.The high data rate of LTE not only demands a wider bandwidth but also a more advanced modulation technique. OFDM was considered as an optimum solution for downlink transmission requirement. But this modulation scheme has some major limitations like high PAPR which can increase complexity and power of transmitter (mobile) in uplink. So an uplink scheme should be considered with trade-off between low computation complexity and system performance. A solution to this problem was to use SC-FDMA which is similar to OFDM but has an additional DFT pre-coding prior to OFDM modulation.

The survey is organized as follows. Section 2 provides a brief overview on OFDM with its advantages and limitations. Section 3 discusses the various PAPR reduction techniques. Section 4 provides a detailed explanation on SC-FDMA. Section 5 discusses the simulation results obtained from different papers. The survey is finally concluded with highlights on the main observations made in the study.

\section{OFDM}

The transmission bandwidth required for communication has increased for taking care of increasing demand for higher data rates. A wide transmission bandwidth can increase frequency selectivity of the channel and thus inter-symbol interference
(ISI) becomes a major issue. An efficient way to mitigate frequency-selective fading of wide band channel is to use multicarrier modulation, which divides the entire channel into a number of smaller sub-channels. Each sub-channel will undergo a flat fading since the signal bandwidth is narrower than coherence bandwidth of the channel.

Orthogonal frequency division multiplexing is a special case of frequency division multiplexing where sub-carriers are orthogonal to one another. This allows overlapping of subcarriers which can ensure efficient bandwidth utilization compared to conventional frequency division multiplexing which requires guard bands between adjacent sub-bands. Orthogonality of sub-carriers is achieved by using inversediscrete Fourier transformation. Thus discrete Fourier transform at transmitter and its inverse at receiver form the signal processing techniques at the heart of OFDM implementations [1]. OFDM is basically a combination of multiplexing and modulation. In OFDM the signals are first split into independent channels modulated by data and then remultiplexed to create OFDM carrier. Since maximum of a subcarrier corresponds to zeros of another subcarrier, each subcarrier can be demodulated independently of the other.

The various advantages of OFDM includes

- Tolerance to multi-path delay spread

- Robustness to channel distortion

- Throughput maximization

- Effective bandwidth utilization

- Frequency Diversity

OFDM can ensure a wide range of advantages in wireless communication. But it has some major limitations. 


\subsection{Limitations of OFDM}

\subsubsection{Peak to Average Power Ratio}

OFDM signal is vector sum of a number of sub-carriers with different phases. At some time instances, this sum will be large and at other times it may be small. As a result the peak value of the signal will be substantially larger than the average value. The complex OFDM signal can be represented by

$$
x(t)=1 / N \sum_{n=0}^{N-1} A_{n}(t) e^{j\left(\omega_{n} t+\phi_{n}(t)\right)}
$$

Where $\mathrm{N}$ is the number of subcarriers, $A_{n}(t)$ is amplitude of each sub-carrier, $\omega=2 \pi_{\mathrm{f}}$ and ${ }^{\phi_{n}(t)}$ is phase of each subcarrier. The high envelope fluctuation in signal amplitude can result in signal distortion which can result in out-of band spectral re-growth of the signals. To accommodate the high PAPR signal, the linear range of high power amplifier has to be increased [2]. It can in turn increase the complexity and cost of high power amplifiers. PAPR of a signal $x(t)$ is given by the following equation

$$
P A P R=\frac{\max |x(t)|^{2}}{E\left\{|x(t)|^{2}\right\}}
$$

The High PAPR value can also reduce the efficiency of HPA because efficiency is given by,

$$
\text { Efficiency }=\frac{0.5}{P A P R}
$$

The PAPR problem can be reduced by providing a back-off in input signal but it will reduce efficiency because HPA will not be able achieve maximum efficiency when operating below saturation point. Analog to digital converters are also affected by this factor since quantization error will increase with high PAPR.

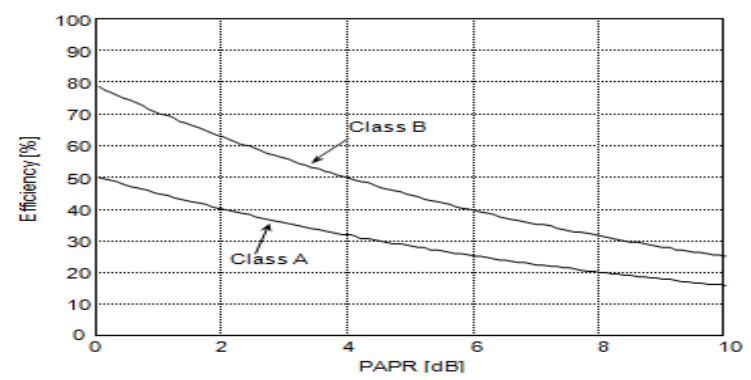

Fig-1: A theoretical relationship between PAPR and transmit power efficiency for ideal High power amplifier [7]

\subsubsection{Carrier Frequency Offset}

OFDM signal consist of a number of narrow sub-carriers which require high degree of frequency synchronization because an offset in carrier frequency can destroy the orthogonality of sub-carriers which can in turn increase multiple access interference among users.

\subsubsection{Sensitivity to Spectral Null}

The OFDM signal detection is done in frequency domain so it is highly sensitive to spectral null. It requires an adaptive coding scheme to overcome spectral nulls in the channel.

\section{PAPR REDUCTION TECHNIQUES}

In uplink communication PAPR becomes a major constraint in the design of mobile terminals. PAPR affects uplink communication than downlink because compared to base station we cannot increase the complexity and power of mobile terminals. So a number of PAPR reduction techniques were developed. PAPR reduction techniques can be classified into two:

\subsection{Distortion Based Techniques}

Distortion based technique will cause spectral re-growth of signals. It is one of the most straightforward PAPR reduction methods. Clipping is a distortion based technique in which clipper limits the peak of the input OFDM signal to a predetermined threshold value. But this is a non-linear operation and can result in out-of band radiation of signals and degradation in bit error rate performance of the system.

To overcome these limitations repeated clipping and filtering of signals to several times can reduce out of-band radiation and PAPR of signals to desired level [3]. But this technique cannot eliminate in-band radiation of the signal.

\subsection{Non-distortion Based Techniques}

PAPR reduction techniques which do not distort shape and cause any spectral re-growth of OFDM signals are called nondistortion techniques. Tone reservation is one such technique [5] in which a set of unused or reserved tones are selected to design a peak cancelling signal. Since the reserved tones are orthogonal chances of distortion due to additive noises are eliminated. A major drawback of this method is the slow convergence toward optimal solution and it has resulted in bandwidth sacrifice as tones have to be reserved.

Multiple signal representation techniques like selected mapping, partial transmit sequence are distortion less PAPR reduction techniques [4].In selected mapping input data is multiplied with a random series and resultant series with lowest PAPR is chosen for transmission. This method has the advantage that it doesn't eliminate peaks and can handle any number of subcarriers. But the limitation of this technique is 
that it requires additional side information that requires to be transmitted to the receiver of the system in order to recover information. Partial transmit sequence is flexible and effective for OFDM system. In this method the input data frame is divided into non-overlapping sub blocks and each sub block is phase shifted by a constant factor to reduce PAPR. All these techniques can provide PAPR reduction but are iterative in nature. So the OFDM transmitter complexity will increase with number of iterations.

An alternate approach to overcome PAPR problem is based on signal transformation. This technique involves a signal transformation prior to amplification at transmitter and an inverse transformation prior to demodulation at the receiver. Some of the transformations include companding, phase modulation and discrete cosine transformation. Conversion of OFDM signal into constant envelope by phase modulation is a technique which can ensure a lowest achievable PAPR of $0 \mathrm{~dB}$. It can provide a faster side-lobe roll-off than OFDM. But a major limitation [6] of this technique was orthogonality of subcarriers was not being maintained.

The major reason for PAPR in OFDM is due to its multicarrier structure. So conversion of multi-carrier OFDM signal into single carrier can effectively reduce PAPR of the signals [7]. Pre-coding of OFDMA signal with Discrete Fourier transformation can convert it into single carrier frequency division multiple access signal (SC-FDMA). Thus SC-FDMA can overcome PAPR problem of OFDMA and many of its limitations like sensitivity to carrier frequency offset, spectral nulls (data detection is done in time domain) and can also reduce complexity of transmitter.

\section{SC-FDMA}

Single carrier frequency division multiple access utilizes single carrier modulation at the transmitter and frequency domain equalization at the receiver. It has similar performance and essentially the same overall complexity as that of an OFDMA system [8-9].SC-FDMA can provide flexible bandwidth assignment for different users and was adopted for uplink in 3GPP Long Term Evolution (LTE) due to its low PAPR property. The structure of SC-FDMA transmitter and receiver are given below

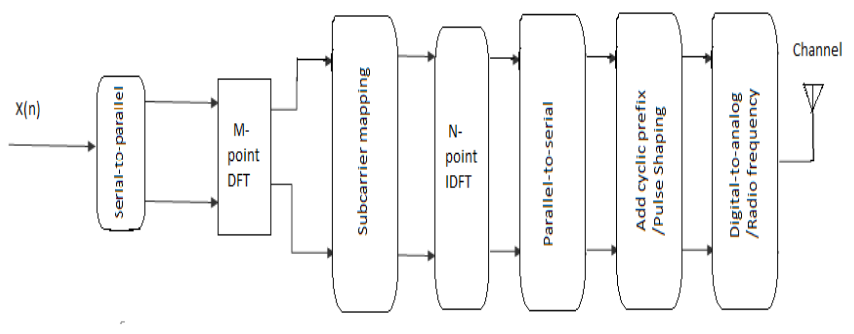

Fig-2: SC-FDMA Transmitter [7]

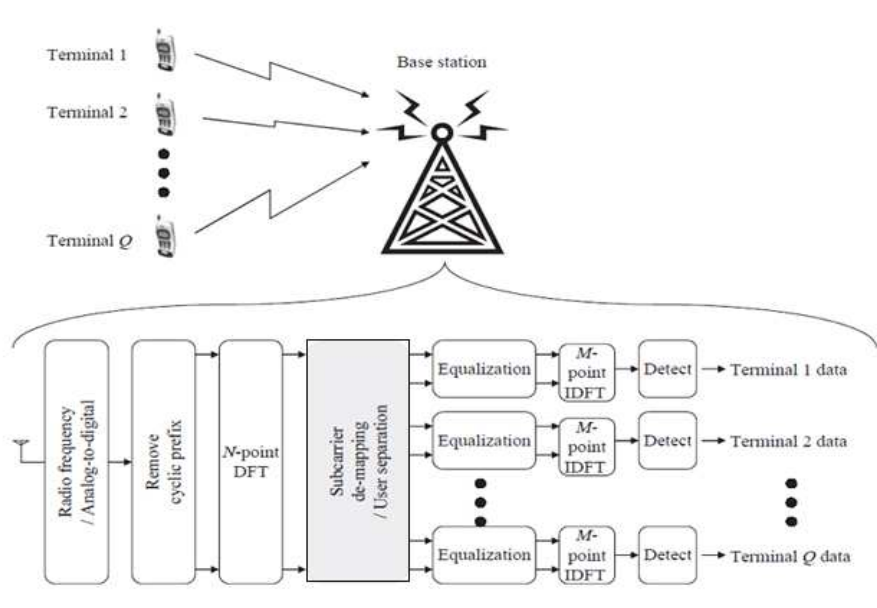

Fig-3: SC-FDMA Receiver [7]

The information bits can be modulated using any modulation techniques like QPSK, QAM or CPM. The aim of a modulation scheme is not only to transmit message signals through a radio channel but also to achieve this function with best quality, power efficiency and minimum bandwidth. Constant envelope modulation like QPSK can ensure high power efficiency and can maintains low spectral side lobes. Modulation scheme like CPM have constant envelope and continuous phase which can provide high spectral efficiency and better PAPR reduction. The modulation index can control the spectral confinement of CPM signal, smaller the index better is the spectral confinement. When modulation index increases BER performance will be improved. But the modulation and demodulation of CPM is complicated by the fact that the initial phase of each symbol is determined by the cumulative phase of all previous transmitted symbols which requires the receiver to have memory. The PAPR value varies with the modulation schemes used.

The complex modulated signal then undergoes discrete time Fourier transformation. The number of DFT points $(\mathrm{M})$ is determined by the number of symbols to be transmitted. The DFT output is then mapped on different sub-carrier. The number of sub-carriers $(\mathrm{N})$ is given by the following equation

$$
\mathrm{N}=\mathrm{M} \cdot \mathrm{Q}
$$

Where, $\mathrm{M}$ represents the number of DFT points and Q represents number of users.

Sub-carrier mapping can be divided into two. They are

1) Localized Mapping (LFDMA) and

2) Distributed Mapping.

In localized mapping each terminal is provided a set of adjacent sub-carriers. LFDMA can potentially achieve multiuser diversity in the presence of frequency selective fading by 
assigning each user to subcarriers in a portion of the signal band where that user has favorable transmission characteristics (high channel gain)[12].This demands channel-dependent scheduling (CDS) of subcarriers. CDS requires the system (base station) to monitor the channel quality as a function of frequency for each terminal, and adapt subcarrier assignments according to changes in channel frequency responses of all terminals. In distributed mapping each terminal is provided a set of sub-carriers that are distributed over the entire bandwidth .This can ensure frequency diversity. Interleaved sub-carrier mapping (IFDMA) is a special case of distributed mapping where sub-carriers allocated for a user are equidistant to one another. The time domain symbols of IFDMA [11] are a simple repetition of input time symbols scaled by a factor (1/Q).The time domain symbols of LFDMA have exact copies of input time symbols in $\mathrm{M}$ multiple sample positions. It was found that a system having many users with each transmitting at a moderate bit rate is better off with IFDMA, while LFDMA can work better in a system with high-bit-rate users. Localized mapping are generally used in LTE uplink applications.

The transmitter performs an important signal processing operation before transmission. It inserts symbols referred to as a cyclic prefix (CP) to provide guard time to prevent interblock interference (IBI) arising due to multipath propagation. If the length of the $\mathrm{CP}$ is longer than the maximum delay spread of the channel, or is equal to length of channel impulse response, there will be no IBI. CP is a copy of the last portion of the information block. It can convert discrete time linear convolution into discrete time circular convolution. Thus data transmitted through the channel can be modeled as a circular convolution between channel impulse response and transmitted data block, which in frequency domain is a pointwise multiplication of the same. Thus the complexity of equalization at the receiver can be reduced.

The transmitter performs a linear filtering operation referred to as pulse shaping for transmission of signal through band limited channel [13]. Raised-cosine filters (RC) are widely used for pulse shaping in wireless communication systems. As roll off factor of filter increase PAPR reduces significantly. But a trade-off between PAPR and out of-band radiation is required because out-of-band radiation can increase with increase in roll-off. The performance of OFDM and different sub carrier mapping of SC-FDMA were compared [14].It was observed that PAPR was less when no pulse shaping was used for IFDMA and LFDMA signals. But PAPR increases significantly for IFDMA when raised cosine pulse shaping was used and for LFDMA PAPR hardly increases with pulse shaping. When pulse shaping was carried out using Rootraised cosine filter (RRC), it was found that RRC pulse shaping filter was effective in reducing PAPR than RC pulse shaping filter. The table below gives the numerical analysis of PAPR for different modulation schemes for IFDMA and LFDMA for raised cosine filer with different roll off $(\alpha)$.
Table- 1: Comparison of PAPR for Different Modulation Schemes and Filter Roll-Off [14]

\begin{tabular}{|c|c|c|c|c|c|c|c|}
\hline \multirow[t]{2}{*}{ Modulation } & \multicolumn{3}{|c|}{ IFDMA } & \multicolumn{3}{|c|}{ LFDMA } & \multirow[b]{2}{*}{ OFDMA } \\
\hline & $\begin{array}{l}\text { No } \\
\text { Pulse } \\
\text { Shaping }\end{array}$ & $\begin{array}{l}\text { Pulse } \\
\text { shaping } \\
(a=0.5)\end{array}$ & $\begin{array}{l}\text { Pulse } \\
\text { shaping } \\
(\alpha=0.22)\end{array}$ & $\begin{array}{l}\text { No } \\
\text { Pulse } \\
\text { Shaping }\end{array}$ & $\begin{array}{l}\text { Pulse } \\
\text { shaping } \\
(\alpha=0.5)\end{array}$ & $\begin{array}{l}\text { Pulse } \\
\text { shaping } \\
(a=0.22)\end{array}$ & \\
\hline QPSK & $O d B$ & $4.3 \mathrm{~dB}$ & $6.1 \mathrm{~dB}$ & $7.5 \mathrm{~dB}$ & $7.6 \mathrm{~dB}$ & $7.6 \mathrm{~dB}$ & $10.7 \mathrm{~dB}$ \\
\hline 8PSK & $O \mathrm{~dB}$ & $4.2 \mathrm{~dB}$ & $5.9 \mathrm{~dB}$ & $7.4 \mathrm{~dB}$ & $7.5 \mathrm{~dB}$ & $7.5 \mathrm{~dB}$ & $10.6 \mathrm{~dB}$ \\
\hline 16QAM & $3.5 \mathrm{~dB}$ & $6.6 \mathrm{~dB}$ & $7.7 \mathrm{~dB}$ & $8.4 \mathrm{~dB}$ & $8.4 \mathrm{~dB}$ & $8.5 \mathrm{~dB}$ & $10.5 \mathrm{~dB}$ \\
\hline 32QAM & $3.4 \mathrm{~dB}$ & $6.4 \mathrm{~dB}$ & $7.5 \mathrm{~dB}$ & $8.2 \mathrm{~dB}$ & $8.3 \mathrm{~dB}$ & $8.4 \mathrm{~dB}$ & $10.6 \mathrm{~dB}$ \\
\hline 64-QAM & $4.8 \mathrm{~dB}$ & $7.1 \mathrm{~dB}$ & $8.0 \mathrm{~dB}$ & $8.6 \mathrm{~dB}$ & $8.7 \mathrm{~dB}$ & $8.7 \mathrm{~dB}$ & $10.5 \mathrm{~dB}$ \\
\hline
\end{tabular}

In an SC-FDMA receiver the reverse operation of transmitter is carried out. The receiver transforms the signal into frequency domain via DFT, de-maps the subcarriers and then performs frequency domain equalization. The equalization process is done in frequency domain because in broadband channels conventional time domain equalizers are impractical as they have a very long channel impulse response in time domain. In cellular systems with severe multipath propagation SC-FDMA signals arrives the base station with substantial inter-symbol interference. The base station uses an adaptive frequency domain equalization to cancel this interference. Most of the well-known time domain equalization techniques such as minimum mean square error (MMSE) equalization, decision feedback equalization (DFE) and turbo equalization can be used. The equalized symbols are transformed back to time domain via IDFT and then detection and decoding will take place in the time domain.

In LTE uplink transmission it was observed [15] that OFDMA turns out to have better performance with high-order modulations and are typically used for users near base station having favorable propagation conditions. SC-FDMA can provide better performance with QPSK and lower code rates and are used near cell edges for users with bad propagation conditions. So OFDMA can provide higher cell capacity and SC-FDMA can lead to cell range extension.

Spreading in SC-FDMA using code-division multiple access (SC-FDMA-CDMA) can provide better PAPR reduction than OFDMA-CDMA [16]. It can combine both features of SCFDMA and Direct sequence CDMA. Single carrier nature of SC-FDMA signal can effectively reduce PAPR while spreading of signals can ensure easy frequency planning, high immunity against interference and flexible data rate adaptation.SC-FDMA-CDMA can ensure both IFDMA and LFDMA mapping to co-exist (Hybrid mapping) which can ensure higher data throughput than conventional sub-carrier mapping techniques. But hybrid sub-carrier mapping can destroy orthogonality of users which can be overcome by using channel dependent scheduling and adaptive modulation. The spreading code length is defined by DFT size and the 
number of spreading code will define the number of active users in the system. It was observed that increase in spreading gain can provide better BER performance for the system.

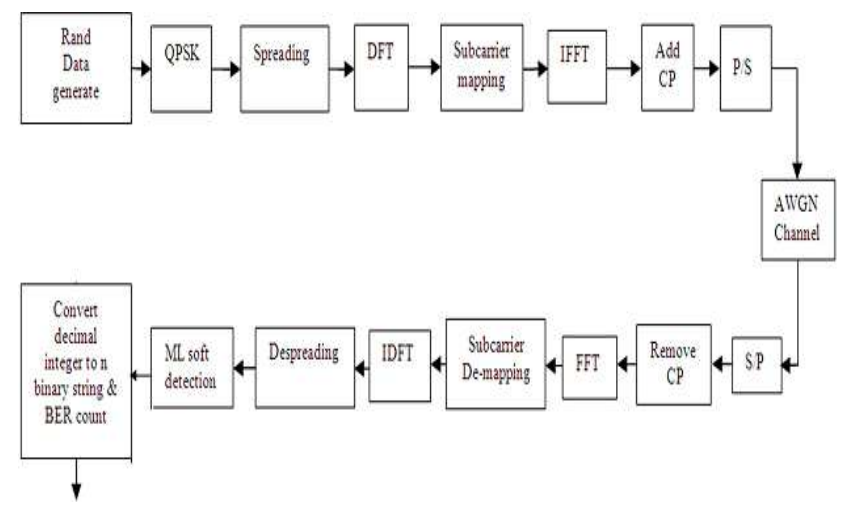

Fig-4: SC-FDMA-CDMA System [17]

\section{SIMULATION RESULTS}

The simulation of SC-FDMA system for QPSK and 16-QAM modulation was carried out for the following parameters.

Table-2: Simulation Parameters

\begin{tabular}{|l|l|}
\hline No of Symbol Block & 100000 \\
\hline DFT Size & 64 \\
\hline IFFT Size & 256 \\
\hline No of users & 4 \\
\hline Sub-carrier Frequency & $15 \mathrm{Khz}$ \\
\hline Filter & Raised Cosine \\
\hline Roll -Off & 0.5 \\
\hline Oversampling Factor & 8 \\
\hline
\end{tabular}

The complementary cumulative distribution (CCDF) function Vs PAPR plot of IFDMA and LFDMA was compared with OFDMA. It was observed that SC-FDMA signal has significant PAPR reduction compared to OFDMA and PAPR of IFDMA was lower than LFDMA.

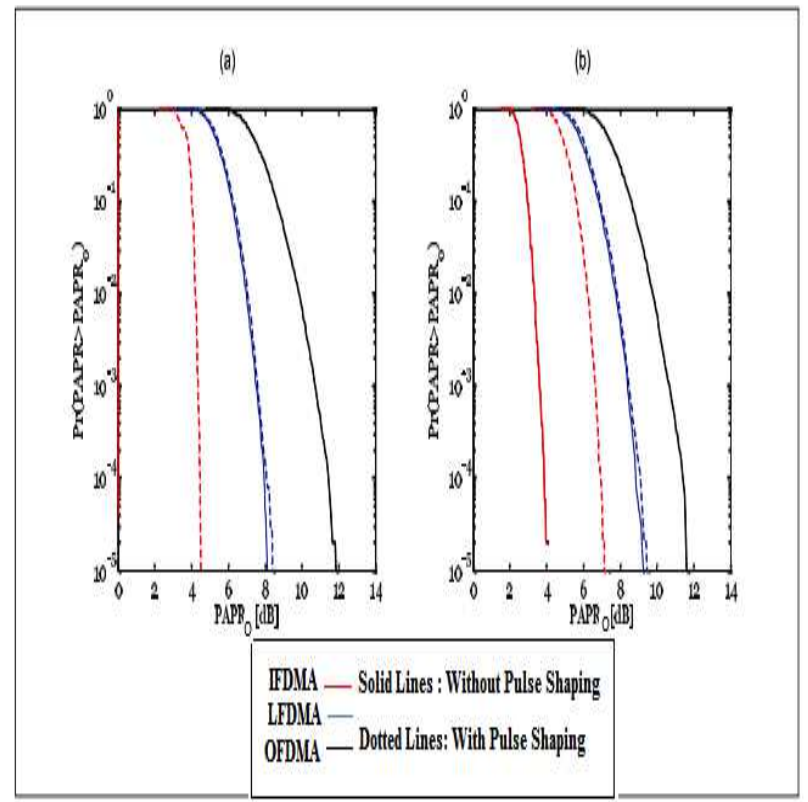

Fig-5: CCDF Vs PAPR of IFDMA, LFDMA and OFDMA signals for (a) QPSK and (b) 16- QAM [9]

The CCDF Vs PAPR plot of IFDMA, LFDMA and OFDMA for different roll-off of raised cosine filter was compared. It was observed that PAPR will reduce with increase in filter roll-off.

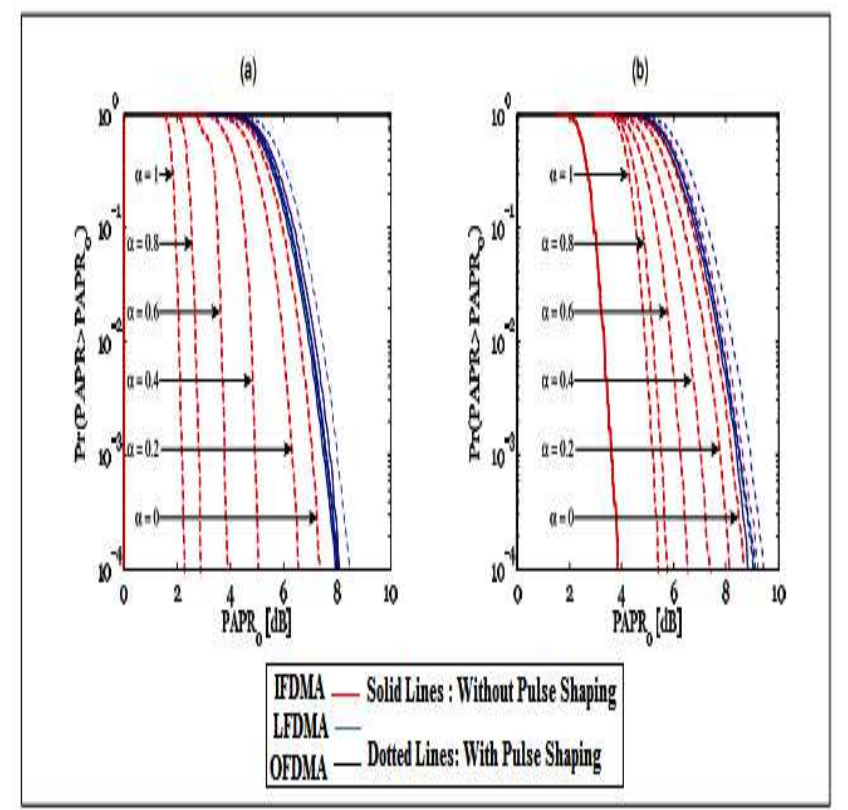

Fig- 6: CCDF Vs PAPR of IFDMA, LFDMA and OFDMA signals for different roll-off (a) QPSK and (b) 16-QAM [9] 
The PAPR performance of SC-FDMA-CDMA and OFDMCDMA was compared for a frame length of 100, Spread length of 16, for 8 users with 1000 data blocks .The PAPR of SC-FDMA-CDMA was found to be lower than OFDMCDMA

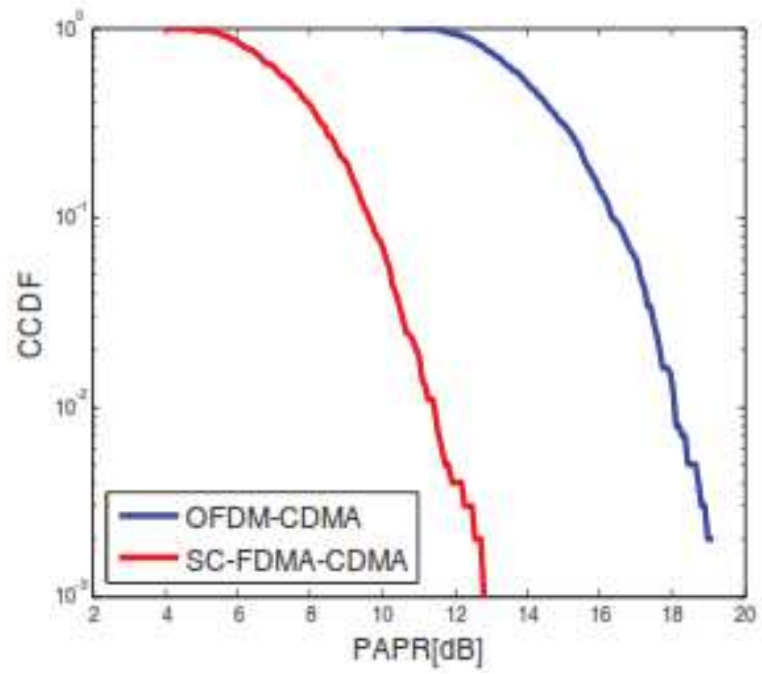

Fig-7: PAPR performance of SC-FDMA-CDMA and OFDMCDMA [16]

\section{CONCLUSIONS}

In this paper we have done a survey on various modulation schemes to determine their suitability in high data rate uplink communication systems and it was found that SC-FDMA was a better choice. SC-FDMA can provide better PAPR reduction than OFDMA and can overcome many of its limitations. Besides uplink communication SC-FDMA is used in various applications like return-link of interactive broadband systems, aeronautical telemetry, land mobile satellite communication systems etc.SC-FDMA can also be used for multiuser communication in downlink for obtaining a more energy efficient and greener base station. It was also observed that PAPR of signal will vary with the modulation used and a trade-off between PAPR and out-of band signal energy has to be considered while choosing the roll-off factor for pulse shaping. Besides this, spreading in SC-FDMA can provide both features of SC-FDMA and CDMA which has better advantages than OFDM-CDMA.

\section{REFERENCES}

[1]. Ramjee Prasad, "OFDM for wireless communication systems", Artech House, Inc.2004

[2]. Mruthyunjaya, "Effect of Peak-to-Average Power Ratio Reduction on the Multicarrier Communication System Performance Parameters", International Journal of Electrical and Computer Engineering, 2009
[3]. J.Armstrong, "Peak-to-average power reduction for OFDM by repeated clipping and frequency domain filtering,"IEEE Electron. Lett, vol. 38, pp. 246-247, Feb. 2002 [4]. A. D. S. Jayalath and C. R. N. Athaudage, "On the PAR Reduction of OFDM signals using multiple signal representation,"IEEE commun. Lett, vol. 8, pp. 425- 427, July 2004

[5]. Brian S. Krongold, and Douglas L. Jones, "An ActiveSet Approach for OFDM PAR reduction via Tone Reservation", IEEE Transactions on Signal Processing, Vol. 52, No. 2, February 2004

[6]. Steve C. Thompson, Ahsen U. Ahmed, John G. Proakis, James R. Zeidler and Michael J. Geile, "Constant Envelope OFDM", IEEE Transactions on Communications, Vol. 56, No. 8, August 2008

[7]. Hyung.G. Myung and David. Goodman, "Single carrier FDMA - A new air interface for long term Evolution", Wiley Publications, 2008

[8]. Marilynn P. Wylie-Green, Erik Perrins and Tommy Svensson, "Introduction to CPM-SC-FDMA: A Novel Multiple-AccessPower-Efficient Transmission Scheme", 2011 IEEE

[9]. Hyung G. Myung, Junsung Lim, and David J. Goodman, "Single Carrier FDMA for uplink wireless transmission",IEEE Vehicular Technology Magazine, September 2006

[10]. Wafaa Radi, Hesham ElBadawy, Salwa ElRamly, "Peak to Average Power Ratio Reduction Techniques for Long Term Evolution- Single Carrier Frequency Division Multiple Access System," International Journal of Advanced Engineering Sciences and Technologies, Vol No. 6, Issue No. 2, $230-236,2011$

[11]. Mohamed Salah, Gamal Abdel-Fadeel and Zaki B. Nossair, "Peak to Average Power Ratio Reduction in Single Carrier OFDMA Systems", 13th International Conference on Aerospace Sciences \& Aviation Technology ASAT- 13, May $26-28,2009$

[12]. Hyung G. Myung, "Introduction to single carrier FDMA", 15th European Signal Processing Conference (EUSIPCO 2007), Poznan, Poland, September 3-7, 2007,

[13]. Ken Gentile, "The care and feeding of digital, pulseshaping filters," www.rfdesign.com April 2002

[14]. Hyung G. Myung Junsung Lim David J. Goodman, “ Peak-To-Average Power Ratio of Single carrier Fdma signals with Pulse shaping",17th Annual IEEE International Symposium on Personal, Indoor and Mobile Radio Communication, 2006

[15]. Christina Ciochina and Hikmet Sari, "A review of OFDMA and Single-carrier FDMA and some recent results, In Proc IEEE, vol.1, No1, 2010

[16]. Zhongqiang Luo, Xingzhong Xiong, "Performance Comparison of SC-FDMA-CDMA and OFDM-CDMA Systems for uplink", 2011 IEEE

[17]. Dinesh N. Bhange, Pranita P. Dhakulkar, Trushna G.Deotale, "Single Carrier Spread Spectrum Technique for uplink", International Journal of Electronics communication and Computer Engineering Volume 4, Issue (2) REACT-2013 


\section{BIOGRAPHIES}

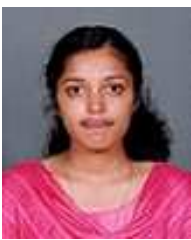

Prittu Ann Thomas has graduated from

Saintgits College of Engineering of Mahatma Gandhi University in Electronics \& Communication Engineering in 2012. She is currently pursuing her M-Tech Degree in Wireless Technology from Toc $\mathrm{H}$ Institute of Science \& Technology, Arakunnam. Her research interest includes Mobile Communication and Digital Communication

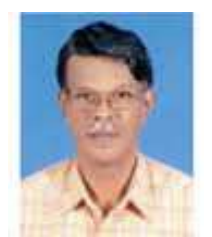

M. Mathurakani has graduated from Alagappa Chettiar College of Engineering and Technology of Madurai University and completed his masters from PSG college of Technology of Madras University. He has worked as a Scientist in Defense Research and development organization (DRDO) in the area of signal processing and embedded system design. He was honoured with the DRDO Scientist of the year award in 2003.Currently he is a professor in Toc $\mathrm{H}$ Institute of Science and Technology, Arakunnam. His area of research interest includes signal processing algorithms, embedded system implementations, reusable architecture and communication systems. 